\title{
Hierarchical Bayesian Algorithm for Diffuse Optical Tomography
}

\author{
Murat Guven, Birsen Yazici \\ Electrical, Computer, and Systems \\ Engineering Department \\ Rensselaer Polytechnic Institute \\ Troy, NY, USA
}

\author{
Xavier Intes , Britton Chance \\ Department of Biophysics and Biochemistry, \\ University of Pennsylvania, \\ Philadelphia, PA, USA
}

\begin{abstract}
Diffuse Optical Tomography (DOT) poses a typical illposed inverse problem with limited number of measurements and inherently low spatial resolution. In this paper, we propose a hierarchical Bayesian approach to improve spatial resolution and quantitative accuracy by using a priori information provided by a secondary high resolution anatomical imaging modality, such as Magnetic Resonance (MR) or X-ray. The proposed hierarchical Bayesian approach allows incorporation of partial a priori knowledge about the noise and unknown optical image models, thereby capturing the function-anatomy correlation effectively. $\mathrm{Nu}$ merical simulations demonstrate that the proposed method avoids undesirable bias towards anatomical prior information and leads to significantly improved spatial resolution and quantitative accuracy.
\end{abstract}

\section{INTRODUCTION}

Diffuse Optical Tomography (DOT) is a non-invasive imaging modality that makes use of the light in the NearInfrared (NIR) spectrum [1]. The inverse problem in DOT involves reconstruction of spatially varying absorption and scattering properties as well as fluorophore lifetime and yield in tissues from boundary measurements. These fundamental quantities can be utilized to obtain tissue oxyand deoxyhemoglobin concentrations, blood oxygen saturation, water, fat, and contrast agent uptake in tissue [2]. The unique physiological and biochemical information offered by DOT is very valuable for practical applications such as breast cancer diagnosis [3], cognitive activity monitoring [4], brain tumor and hemorrhage detection [5] with a growing list of applications in fluorescence tomographic imaging [6].

Recently several research groups reported development of hybrid imaging systems combining optical methods with high resolution anatomical imaging techniques. These in- clude a concurrent X-ray tomosynthesis-DOT system at Massachusetts General Hospital [7], MRI-DOT/DOS (Diffuse Optical Spectroscopy) systems at University of Pennsylvania [8], University of California at Irvine [9] and Dartmouth College [10]. These multi-modality developments are all motivated by the fact that DOT offers unique functional information (such as tissue oxy- and deoxyhemoglobin concentrations) while high resolution anatomical imaging modalities provide complementary information for disease diagnosis and understanding with superior localization and spatial resolution. A number of studies have shown that use of a priori high resolution anatomical images leads to improved diffuse optical image reconstruction [11], [12], [13], [7], [10] as well as improved spatial resolution.

In all the studies referenced above, the performance of the DOT image reconstruction relies on the assumption that the correlation between the anatomical and optical images is high. However, there may be regions in the optical image that do not have any anatomical counterparts. As a result, the assumption of strong optical-anatomy correlation may cause undesirable, erroneous bias in optical image reconstruction. The hierarchical Bayesian framework affords such a flexibility in designing prior image and noise models to address the optical-anatomy correlation.

In this paper, we consider a two-level hierarchical Bayesian formulation to incorporate a priori anatomical and tissue classification information into the DOT image reconstruction. We start with the segmentation of the high resolution image and classify the segmented image into subimages representing major tissue types. Based on the tissue label information extracted from the anatomical image, we design the first stage of the prior distribution on the unknown optical image as a function of unknown hyperparameters, namely the image mean and standard deviation. Next, we design the parametric noise model with an associated unknown hyperparameter, which is related to the noise variance. The uncertainty in the models owing to the unknown hyperparameters is addressed by defining hyperpriors on the 
hyperparameters, which constitutes the second stage of the hierarchical formulation. The hyperpriors on the hyperparameters of the image model are formulated with the aid of coregistered tissue classification. We refer to sections 2 and 3 for a detailed discussion of the hierarchical noise and image models.

Having designed the hierarchical noise and image models, we formulate the joint distribution of the measurements, the image and the unknown hyperparameters associated with the noise and image models. In order to estimate the hyperparameters, we adapt the linear conjugate gradient (CG) algorithm to include a hyperparameter estimation step followed by an image update. In this context, we apply an iterative empirical Bayesian approach [14] to estimate the hyperparameters, which in turn gives the maximum a posteriori (MAP) estimates of the hyperparameters at each $\mathrm{CG}$ iteration prior to the image update.

We perform simulation experiments to evaluate the performance of the proposed hierarchical Bayesian formulation and hyperparameter estimation scheme. Our results indicate that hierarchical Bayesian approach captures the function-anatomy correlation properly and provides improved DOT image reconstruction without introducing undesirable bias towards a priori anatomical information. Our simulation experiments show that the proposed method provides accurate reconstruction of tumors even when tumor contrast is absent in the anatomical image.

The paper is organized as follows: Section 2 defines the forward model. Section 3 provides background on the hierarchical Bayesian formulation of the inverse problem and describes the components of the hierarchical model. Section 4 presents the simultaneous estimation of the optical image and the unknown hyperparameters. Section 5 includes numerical experiments to validate the properties of the proposed approach. Section 6 summarizes our results and conclusion.

\section{Forward Model}

In this work, we focus on the reconstruction of absorption coefficients of the medium. Therefore, we assume that the diffusion coefficient of the medium is known and spatially invariant. As a result, the following diffusion equation given in frequency domain suffices to define the forward model:

$$
D \nabla^{2} \phi(\mathbf{r})-\mu_{a}(\mathbf{r}) \phi(\mathbf{r})-\frac{i \omega}{c} \phi(\mathbf{r})=-A \delta\left(\mathbf{r}-\mathbf{r}_{s}\right),
$$

where $\phi(\mathbf{r})$ represents the spatially varying optical field in the medium $\Omega \subset \mathbb{R}^{3}$, due to the point source $A \delta\left(\mathbf{r}-\mathbf{r}_{s}\right)$ located at $\mathbf{r}=\mathbf{r}_{s}$. $\omega$ denotes the modulation frequency of the source, $c$ is the speed of light and $i=\sqrt{-1}$. $D$ is the diffusion coefficient and $\mu_{a}(\mathbf{r})$ stands for the spatially varying absorption coefficient of the medium.
We have employed the perturbation approach with a first order Rytov approximation to solve the forward problem in the frequency domain to yield a system of linear equations after the discretization of the medium $\Omega$ into $N$ uniform voxels [15]:

$$
\mathbf{y}=\mathbf{W x}+\boldsymbol{\zeta},
$$

where $\mathbf{y}$ is the measurement vector, $\mathbf{W}$ is the Jacobian based on the Rytov approximation computed around a specified homogeneous background $\mu_{a}(\mathbf{r})=\mu_{a 0} . \mathbf{x} \in \mathbb{R}^{N}$ denotes the vector of differential absorption coefficients $\delta \mu_{a}$ of the medium with respect to the homogenous background, and $\zeta$ is the additive noise in the measurement system.

\section{Hierarchical Bayesian Formulation of the Inverse Problem}

We formulate the posterior distribution of the unknown image and compute its maximum a posteriori (MAP) estimate $\hat{\mathbf{x}}_{M A P}$; that is

$$
\hat{\mathbf{x}}_{M A P}=\arg \max _{\mathbf{x}}\{\log p(\mathbf{y} \mid \mathbf{x})+\log p(\mathbf{x} \mid \mathbf{C})\} .
$$

where $p(\mathbf{y} \mid \mathbf{x})$ is the data likelihood, $\log p(\mathbf{x} \mid \mathbf{C})$ is the prior and $\mathbf{C}$ represents the anatomical tissue label information, derived from the a priori anatomical image. In our formulation, the noise statistics and the prior distribution are governed by unknown model parameters $\lambda$ and $\mathcal{Q}$, respectively. Thus, the data likelihood and the image prior in equation (3) are replaced by $p(\mathbf{y} \mid \mathbf{x}, \lambda)$ and $p(\mathbf{x} \mid \mathcal{Q}, \mathbf{C})$, respectively. Here, $\lambda$ is the scalar parameter associated with the noise variance and $\mathcal{Q}$ is the vector of mean and variance of sub-images that correspond to different tissue labels. We will refer to these parameters as hyperparameters. In order to estimate the unknown image and the associated hyperparameters, we consider the joint conditional distribution:

$$
p(\mathbf{y}, \mathbf{x}, \lambda, \mathcal{Q} \mid \mathbf{C})=p(\mathbf{y}, \lambda \mid \mathbf{x}) p(\mathbf{x}, \mathcal{Q} \mid \mathbf{C}) .
$$

Equation (4) can be alternatively expressed as

$$
\begin{array}{r}
\log p(\mathbf{y}, \mathbf{x}, \lambda, \mathcal{Q} \mid \mathbf{C})=\log p(\mathbf{y} \mid \mathbf{x}, \lambda)+\log p(\lambda) \\
+\log p(\mathbf{x} \mid \mathcal{Q}, \mathbf{C})+\log p(\mathcal{Q} \mid \mathbf{C}),
\end{array}
$$

where $p(\lambda)$ is the prior distribution on $\lambda$. We shall refer to $p(\lambda)$ and $p(\mathcal{Q} \mid \mathbf{C})$ as hyperpriors [14]. In this representation, $p(\mathbf{x} \mid \mathcal{Q}, \mathbf{C})$ is the first-stage prior and $p(\mathcal{Q} \mid \mathbf{C})$ stands for the second-stage prior.

\subsection{The Data Likelihood Model}

The measurement vector $\mathbf{y}$ is formed as

$$
\mathbf{y}=\left[\begin{array}{lllllll}
y_{11}^{f_{1}} & y_{12}^{f_{1}} & \cdots & y_{S D}^{f_{1}} & y_{11}^{f_{2}} & \cdots & y_{S D}^{f_{F}}
\end{array}\right]^{T},
$$


where $S$ is the number of sources, $D$ is the number of detectors, and $F$ is the number of frequencies associated with each source. The total number of measurements is then equal to $P=S \times D \times F$. For computational efficiency, we limit the data set to the real part of the measurements, thus $\mathbf{y} \in \mathbb{R}^{P}$.

Consequently, we model the data likelihood in equation (4) as [16]

$$
p(\mathbf{y}, \lambda \mid \mathbf{x})=\frac{1}{K\left|\boldsymbol{\Lambda}_{\zeta}(\lambda)\right|^{1 / 2}} \exp \left[-\frac{1}{2}\|\mathbf{y}-\mathbf{W} \mathbf{x}\|_{\boldsymbol{\Lambda}_{\zeta}^{-1}(\lambda)}^{2}\right],
$$

where we assume a noninformative prior for $\lambda$, which is a uniform density on $\mathbb{R}^{1}$ [14]. In the above distribution, $\boldsymbol{\Lambda}_{\zeta}(\lambda)$ is the covariance matrix of size $P \times P, K$ is the normalization constant and $\|\mathbf{z}\|_{\Lambda}^{2}=\mathbf{z}^{T} \boldsymbol{\Lambda} \mathbf{z}$. Under the assumption of statistical independence, $\boldsymbol{\Lambda}_{\zeta}(\lambda)$ becomes a diagonal matrix with entries $\lambda \sigma_{\zeta_{p}}^{2}$, where $\sigma_{\zeta_{p}}^{2}$ is equal to the absolute value of the $p^{\text {th }}$ measurement and the unknown parameter $\lambda$ controls the scale of the noise covariance matrix [15].

\subsection{Hierarchical Formulation of the Prior Distribution}

We downsample the high resolution anatomical image to match its resolution with that of the optical image. Next, we utilize the downsampled anatomical image to decompose the optical domain into $M$ non-overlapping subimages, each of which is assumed to represent a major tissue type. For instance, for breast, these tissues types can be parenchyma, adipose, and tumor [17]. We assume a Gaussian distribution for each sub-image, with unknown mean and standard deviation. Thus, the first stage in the hierarchical prior distribution for the $i^{t h}$ sub-image is given by

$$
p\left(\mathbf{x}_{i} \mid \boldsymbol{\mu}_{i}, \sigma_{i}\right)=\frac{1}{\left(2 \pi \sigma_{i}^{2}\right)^{N_{i} / 2}} \exp \left[-\frac{1}{2 \sigma_{i}^{2}}\left\|\mathbf{x}_{i}-\boldsymbol{\mu}_{i}\right\|^{2}\right],
$$

for $i=1,2, \ldots, M$ with the implicit assumption that the voxels in each sub-image are statistically independent. $\boldsymbol{\mu}_{i}=\left(\mu_{i} \cdots \mu_{i}\right)^{T}$ is the uniform mean value vector of size $N_{i} \times 1$, where $N_{i}$ stands for the number of voxels in the $\mathrm{i}^{\text {th }}$ sub-image. The covariance matrix associated with the $i^{t h}$ sub-image $\boldsymbol{\Lambda}_{x}\left(\sigma_{i}\right)=\sigma_{i}^{2} \mathbf{I}_{N_{i} \times N_{i}}$ where $\sigma_{i}$ is the standard deviation of each voxel in the $\mathrm{i}^{t h}$ sub-image and $\mathbf{I}_{N_{i} \times N_{i}}$ is the $N_{i} \times N_{i}$ identity matrix. Assuming that the sub-images are statistically independent, the first-stage prior of the image given the tissue label information $\mathbf{C}$ is:

$$
\begin{array}{r}
p(\mathbf{x} \mid \mathcal{Q}, \mathbf{C})=\frac{1}{(2 \pi)^{N / 2}\left|\boldsymbol{\Lambda}_{x}(\boldsymbol{\sigma})\right|^{1 / 2}} \\
\times \exp \left[-\frac{1}{2}\|\mathbf{x}-\boldsymbol{\mu}\|_{\boldsymbol{\Lambda}_{x}^{-1}(\boldsymbol{\sigma})}^{2}\right.
\end{array}
$$

where $\mathcal{Q}=[\boldsymbol{\mu}, \boldsymbol{\sigma}] . \boldsymbol{\mu}$ is the vector of mean values assigned to the sub-images and $\sigma$ is the vector of standard deviations associated with the sub-images, that is

$$
\begin{aligned}
& \boldsymbol{\sigma}=\left[\begin{array}{llll}
\underbrace{\sigma_{1} \cdots \sigma_{1}}_{N_{1}} & \underbrace{\sigma_{2} \cdots \sigma_{2}}_{N_{2}} & \cdots & \underbrace{\sigma_{M} \cdots \sigma_{M}}_{N_{M}}
\end{array}\right]^{T}, \\
& \boldsymbol{\mu}=\left[\begin{array}{llll}
\underbrace{\mu_{1} \cdots \mu_{1}}_{N_{1}} & \underbrace{\mu_{2} \cdots \mu_{2}}_{N_{2}} & \cdots & \underbrace{\mu_{M} \cdots \mu_{M}}_{N_{M}}
\end{array}\right]^{T},
\end{aligned}
$$

and the covariance matrix $\boldsymbol{\Lambda}_{x}(\boldsymbol{\sigma})$ of the image $\mathbf{x}$ is given by:

$$
\left[\begin{array}{ccccc}
\sigma_{1}^{2} \mathbf{I}_{N_{1} \times N_{1}} & 0 & 0 & \cdots & 0 \\
0 & \sigma_{2}^{2} \mathbf{I}_{N_{2} \times N_{2}} & 0 & \cdots & 0 \\
0 & 0 & \ddots & \ddots & 0 \\
\vdots & \ddots & \ddots & \ddots & 0 \\
0 & 0 & 0 & 0 & \sigma_{M}^{2} \mathbf{I}_{N_{M} \times N_{M}}
\end{array}\right] .
$$

The second stage prior involves incorporation of the $a$ priori information into the hierarchical prior distribution; in the form of hyperpriors defined on the unknown hyperparameters of the first stage, that is the mean and standard deviation of different tissue types in the optical image.

We assume a Gaussian distribution for the mean value $\boldsymbol{\mu}$ of the image:

$p(\boldsymbol{\mu} \mid \mathbf{C})=\frac{1}{(2 \pi)^{N / 2}\left|\boldsymbol{\Lambda}_{\mu}(\vartheta)\right|^{1 / 2}} \exp \left[-\frac{1}{2}\|\boldsymbol{\mu}-\tilde{\boldsymbol{\mu}}\|_{\boldsymbol{\Lambda}_{\mu}^{-1}(\vartheta)}^{2}\right]$,

where $\tilde{\boldsymbol{\mu}}=\left.\boldsymbol{\mu}\right|_{\mu_{i}=\tilde{\mu}_{i}}$ and $\tilde{\mu}_{i}$ is the average differential absorption of the $\mathrm{i}^{\text {th }}$ tissue type. $\boldsymbol{\Lambda}_{\mu}(\vartheta)=\left.\boldsymbol{\Lambda}_{x}(\boldsymbol{\sigma})\right|_{\sigma_{i}=\vartheta_{i}}$ is the covariance matrix where $\vartheta_{i}$ stands for the standard deviation of the mean value of each voxel in the $\mathrm{i}^{\text {th }}$ tissue type, for $i=1,2, \ldots, M$.

Similarly, we assume a Gaussian distribution for standard deviation $\sigma$ of the image:

$p(\boldsymbol{\sigma} \mid \mathbf{C})=\frac{1}{(2 \pi)^{N / 2}\left|\boldsymbol{\Lambda}_{\sigma}(\gamma)\right|^{1 / 2}} \exp \left[-\frac{1}{2}\left\|\boldsymbol{\sigma}-\boldsymbol{\mu}_{\sigma}\right\|_{\boldsymbol{\Lambda}_{\sigma}^{-1}(\gamma)}^{2}\right]$,

where $\boldsymbol{\mu}_{\sigma}=\left.\boldsymbol{\mu}\right|_{\mu_{i}=\mu_{\sigma_{i}}}$ and $\boldsymbol{\Lambda}_{\sigma}(\gamma)=\left.\boldsymbol{\Lambda}_{x}(\boldsymbol{\sigma})\right|_{\sigma_{i}=\gamma_{i}}$ for $i=1,2, \ldots, M$. Having designed the first and second stage priors, the hierarchical prior distribution in equation (4) becomes:

$$
\begin{array}{r}
p(\mathbf{x}, \mathcal{Q} \mid \mathbf{C})=p(\mathbf{x} \mid \boldsymbol{\mu}, \boldsymbol{\sigma}, \mathbf{C}) p(\boldsymbol{\mu} \mid \mathbf{C}) p(\boldsymbol{\sigma} \mid \mathbf{C}) \\
=\frac{1}{(2 \pi)^{3 N / 2}\left|\boldsymbol{\Lambda}_{x}(\boldsymbol{\sigma})\right|^{1 / 2}\left|\boldsymbol{\Lambda}_{\mu}(\vartheta)\right|^{1 / 2}\left|\boldsymbol{\Lambda}_{\sigma}(\gamma)\right|^{1 / 2}} \\
\times \exp \left[-\frac{1}{2}\left(\|\mathbf{x}-\boldsymbol{\mu}\|_{\boldsymbol{\Lambda}_{x}^{2}(\boldsymbol{\sigma})}^{2}+\|\boldsymbol{\mu}-\tilde{\boldsymbol{\mu}}\|_{\boldsymbol{\Lambda}_{\mu}^{-1}(\vartheta)}^{2}\right.\right. \\
\left.\left.+\left\|\boldsymbol{\sigma}-\boldsymbol{\mu}_{\sigma}\right\|_{\boldsymbol{\Lambda}_{\sigma}^{-1}(\gamma)}^{2}\right)\right]
\end{array}
$$

In practice, $\tilde{\mu}_{i}$ can be assigned based on the average absorption coefficients of tissue types provided in the literature with a sufficiently large variance $\vartheta_{i}^{2}$. Analogously, the 
mean value of $\boldsymbol{\sigma}$ can be extracted from the error bounds of the average optical properties of tissue types which are documented in the literature. See for example [18] for an exhaustive list of optical coefficients for human tissue and fluids.

\section{Image Reconstruction and Hyperparame- ter Estimation}

Substituting equations (7) and (12) into (4), the joint probability distribution of the measurements, optical image and the hyperparameters is given by

$$
p(\mathbf{y}, \mathbf{x}, \lambda, \boldsymbol{\mu}, \boldsymbol{\sigma} \mid \mathbf{C})=p(\mathbf{y}, \lambda \mid \mathbf{x}) p(\mathbf{x}, \mathcal{Q} \mid \mathbf{C})
$$

Let $\Psi(\mathbf{x}, \lambda, \boldsymbol{\mu}, \boldsymbol{\sigma})$ be the objective functional given by

$$
\Psi(\mathbf{x}, \lambda, \boldsymbol{\mu}, \boldsymbol{\sigma})=-\log p(\mathbf{y}, \mathbf{x}, \lambda, \boldsymbol{\mu}, \boldsymbol{\sigma} \mid \mathbf{C}) .
$$

Then, the image reconstruction becomes an optimization problem in which the objective functional $\Psi(\mathbf{x}, \lambda, \boldsymbol{\mu}, \boldsymbol{\sigma})$ has to be minimized with respect to the image $\mathbf{x}$ and the hyperparameters $\lambda, \boldsymbol{\mu}$ and $\boldsymbol{\sigma}$.

We propose an iterative algorithm based on the empirical Bayesian approach [15] that successively estimates the hyperparameters with only $O(N)$ extra operations at each iteration, where $N$ is the number of voxels. The hyperparameter estimation step is followed by the image update by one iteration of conjugate gradient (CG) algorithm, applied with the current estimates of the hyperparameters.

\section{Results}

We evaluate the proposed method using optical data simulated from an MR-breast image. We show that the a priori information improves the image reconstruction and does not lead to an erroneous bias towards the a priori information.

\subsection{D Experiment with MR-simulated data}

We used the T1-weighted MR breast image from [17] to design a realistic optical breast model (figure 1). The MR breast image was segmented into parenchyma and adipose layers by applying a simple thresholding algorithm with respect to the MR image intensity values. Next, a tumor corresponding to an infiltrating ductal carcinoma revealed by Gd-DTPA (Gadolinium-diethylenetriamine pentaacetic acid) enhancement was inserted (shown in figure 1 as well). Each sub-region was assigned an absorption value as indicated in Ntziachristos (1999) ( $\mu_{a}^{\text {adipose }}=$ $0.03 \mathrm{~cm}^{-1}, \mu_{a}^{\text {parenchyma }}=0.06 \mathrm{~cm}^{-1}, \mu_{a}^{\text {tumor }}=0.09$ $\mathrm{cm}^{-1}$ ) to obtain an initial template (figure 2). To sim-

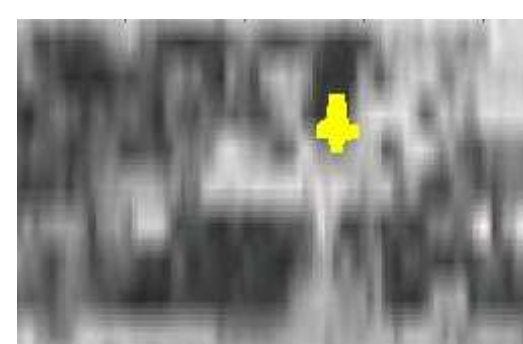

\section{Figure 1. The original MR breast image with an artificial tumor inserted.}

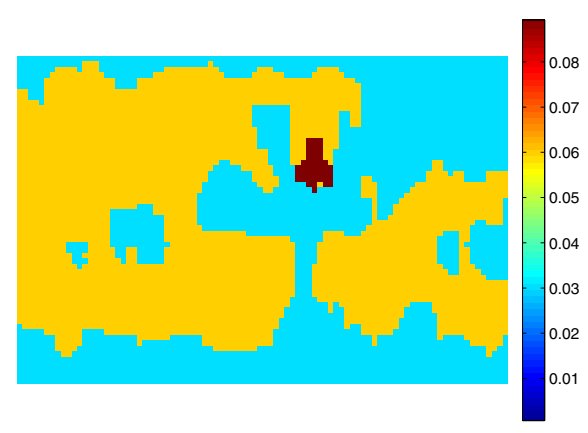

Figure 2. The anatomical template.

ulate a corresponding optical image, zero mean Gaussian noise was added prior to filtering the image by a low-pass filter. The resulting optical image is shown in figure 3 . Note the quantitative and spatial mismatch along the boundaries and especially within the tumor. The homogeneous diffusion coefficient of the medium was set to $0.042 \mathrm{~cm}$. 9 frequencies ranging from 0 to $244 \mathrm{MHz}$ were used to obtain 729 measurements with 9 sources and 9 detectors positioned along $\mathrm{x}$-axis on opposite sides. The optical medium was uniformly discretized into 90 pixels along $\mathrm{x}$-axis and 60 pixels along y-axis leading to a total of $54001 \times 1 \mathrm{~cm}^{2}$ pixels.

We performed two types of experiments to test the performance of the proposed hierarchical Bayesian approach for this problem:

(i) Tumor present both anatomically and optically: In this experiment, the template extracted from the anatomical image shown in figure 2 was used to design the hierarchical image prior. As a result, the optical image was segmented into three sub-images each of which corresponded to the labeled images in the anatomical image as shown in figure 2. 


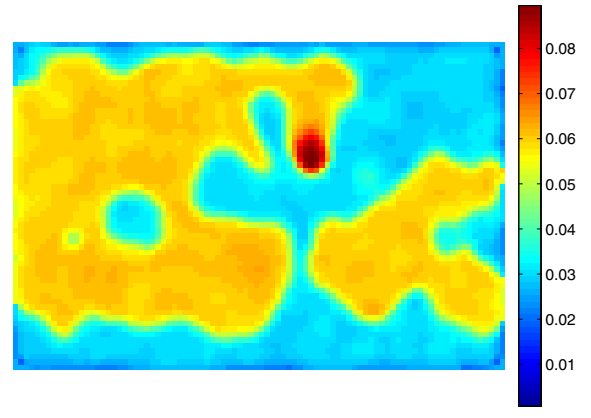

Figure 3. The generated optical image.

In the design of the hyperprior defined on the mean (i.e. $p(\boldsymbol{\mu} \mid \mathbf{C})$ ), values that significantly different than the actual mean of the sub-images were used. Thus, this experiment evaluates the robustness of the proposed method when the true statistics of the optical image are significantly different than the statistics extracted from the prior anatomical image.

The reconstructed image is shown in figure 4. For comparison, the ML estimate of the image is shown in figure 5.

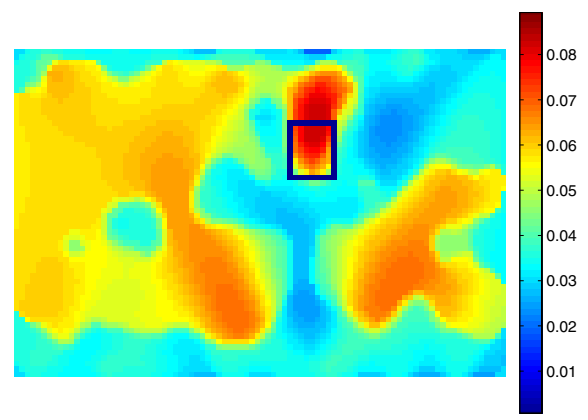

Figure 4. The hierarchical Bayesian reconstruction of the optical image using the anatomical template shown in figure 2 for the design of the hierarchical image model.

(ii) Tumor present optically but not anatomically: In this experiment, we removed the tumor region from the template extracted from the prior anatomical image as shown in figure 6. As a result, the optical image was segmented into two-sub images. The objective of this experiment is to evaluate how well the proposed method reconstructs optical tumors when they are not anatomically present. The reconstructed image for this experiment is given in figure 7 . The set of parameters used in the design of hyperpriors for

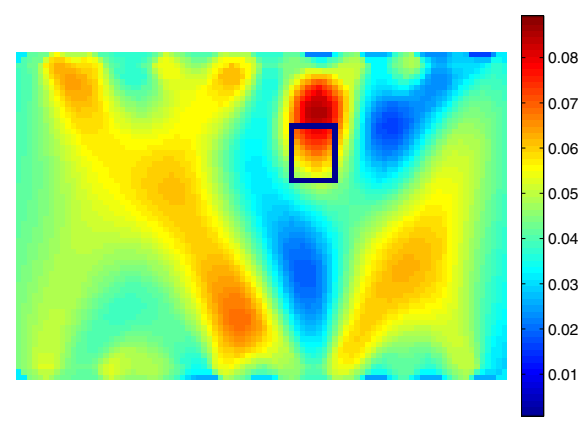

Figure 5. The ML estimate of the image.

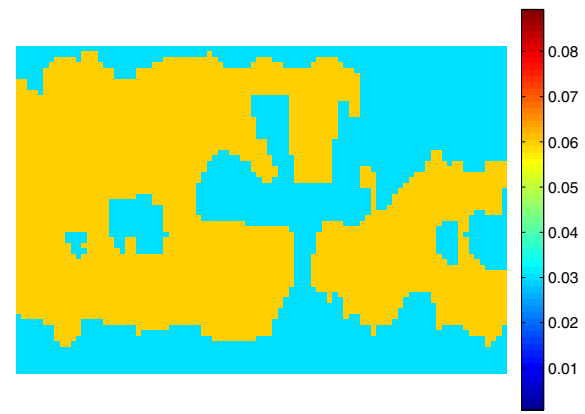

Figure 6. The anatomical template for second experiment. Note that the tumor is not anatomically present.

these experiments and the actual mean of absorption values for each sub-image are shown in table 1.

\section{Conclusion}

In this work we formulated the inverse DOT problem within a hierarchical Bayesian framework where the hierarchical prior distribution is based on the a priori information extracted from a secondary high-resolution anatomical image. Instead of directly constraining the optical image with the anatomical prior, we incorporated the a priori information in the form of hyperpriors to impose constraints on the unknown hyperparameters of the image and noise models. We proposed a computationally efficient iterative algorithm, based on an empirical Bayesian approach, to simultaneously estimate the optical image and the unknown hyperparameters. Our numerical studies show that the hierarchical Bayesian approach provides an effective framework to capture the correlation between optical and anatomical images. 


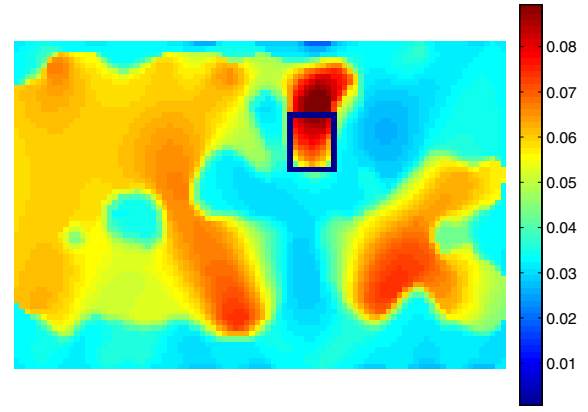

Figure 7. The hierarchical Bayesian reconstruction of the optical image using the anatomical template shown in figure 6 for the design of the hierarchical image model.

\begin{tabular}{|c|c|c|c|}
\hline & Parenchyma & Adipose & Tumor \\
\hline$\left(\tilde{\mu}_{i}+\mu_{a 0}, \vartheta_{i}\right)$ & $(0.038,0.23)$ & $(0.05,0.3)$ & $(0.09,0.54)$ \\
$\left(\mu_{\sigma_{i}}, \gamma_{i}\right)$ & $(0.015,0.228)$ & $(0.02,0.3)$ & $(0.036,0.54)$ \\
\hline$\left(\tilde{\mu}_{i}+\mu_{a 0}, \vartheta_{i}\right)$ & $(0.03,0.18)$ & $(0.06,0.36)$ & N/A \\
$\left(\mu_{\sigma_{i}}, \gamma_{i}\right)$ & $(0.012,0.18)$ & $(0.024,0.36)$ & N/A \\
\hline
\end{tabular}

Table 1. The parameter set used in the first and second experiments. $\mu_{a 0}=0.0439$ for this experiment. N/A stands for "not applicable".

\section{References}

[1] Intes X and Chance B 2005 Non-PET functional imaging techniques: optical, The Radiologic Clinics of North America 43(1) 221-34.

[2] Kincade K 2004 Optical Diagnostics continue migration from bench top to beside Laser Focus World 130-4.

[3] Intes X, Ripoll J, Chen Y, Nioka S, Yodh A and Chance B 2003 In vivo continuous-wave optical breast imaging enhanced with Indocyanine Green Med. Phys. Biol. 30, 1039-47.

[4] Strangman G, Boas D and Sutton J 2002 Non-invasive neuroimaging using near-infrared light Biol. Psychiatry 52 679-93.

[5] Hebden J C, Gibson A, Austin T, Yusof R, Everdell N, Delpy E, Arridge S R, Meek J H and Wyatt J S 2004 Imaging changes in blood volume and oxygenation in the newborn infant brain using three-dimensional optical tomography Phys. Med. Biol.49 1117-30.

[6] Weissleder R and Ntziachristos V 2003 Shedding light onto live molecular targets Nature Medicine 9 123-8.
[7] Li A, Miller E L, Kilmer M E, Brukilacchio T J, Chaves T, Stott J, Zhang Q, Wu T, Chorlton M, Moore R H, Kopans D B and Boas D 2003 A Tomographic Optical Breast imaging guided by 3D mammography Applied Optics 42 5181-90.

[8] Intes X, Yu J, Yodh A G and Chance B 2002 Development and evaluation of a multi-wavelength multi-channel time resolved optical instrument for NIR/MRI mammography co-registration Proc. IEEE 28th Annual Northeast Bioengineering Conf. (IEEE Cat. No.02CH37342) 91-2.

[9] Gulsen G, Birgul O and Nalcioglu O Hybrid DOT and MRI system 2003 Hybrid DOT and MR System Proc. SPIE Optical Tomography and Spectroscopy of Tissue $4955246-52$.

[10] Brooksby B, Dehghani H, Pogue B and Paulsen K D 2003 Near-Infrared (NIR) Tomography Breast Reconstruction with a priori Structural information from MRI: Algorithm Development Reconstructing Heterogeneities IEEE J. Selected Topics in Quantum Electronics 199209.

[11] Dehghani H Pogue B W and Paulsen K D 2002 Development of hybrid NIR/MRI imaging system algorithm: Use of a-priori information for tumor detection in the female breast Proceedings IEEE Int. Symp. on Biomedical Imaging 657-60.

[12] Ntziachristos V 2002 MRI-guided Diffuse Optical Spectroscopy of malignant and benign breast legions Neoplasia 4 347-54.

[13] Xu H, Dehghani H, Pogue B W, Paulsen K D and Dunn J D 2002 Hybrid MR/Near Infrared Imaging of the Muribe Brain: Optimization of optical fiber arrangement and use of a priori knowledge Proceedings IEEE Int. Symp. on Biomedical Imaging 74-7.

[14] Berger J O 1988 Statistical Decision Theory and Bayesian Analysis (Springer Verlag).

[15] Guven M, Yazici B, Intes X and Chance B 2005 Diffuse optical tomography with a priori information Phys. Med. Biol. 50 2837-58.

[16] Ye J C, Bouman C A, Webb K J and Millane R P 2001 Nonlinear Multigrid Algorithms for Bayesian Optical Diffusion Tomography IEEE Trans. on Image Processing 10 909-22.

[17] Ntziachristos V, Chance B and Yodh A G 1999 Differential diffuse optical tomography Optics Express 5 23042.

[18] Mobley J and Vo-Dinh T 2003 Biomedical Photonics, Handbook-Chapter 2 (CRC Press). 